\title{
Structural Reliability Analysis Using Orthogonalizable Power Polynomial Basis Vector
}

\author{
Yejun Li and Bin Huang \\ School of Civil Engineering and Architecture, Wuhan University of Technology, Wuhan, China
}

\begin{abstract}
A new method for structural reliability analysis using orthogonalizable power polynomial basis vector is presented. Firstly, a power polynomial basis vector is adopted to express the initial series solution of structural response, which is determined by a series of deterministic recursive equation based on perturbation technique, and then transferred to be a set of orthogonalizable power polynomial basis vector using the orthogonalization technique. By conducting Garlekin projection, an accelerating factor vector of the orthogonalizable power polynomial expansion is determined by solving small scale algebraic equations. Numerical results of a continuous bridge structure on reliability analysis shows that the proposed method can achieve the accuracy of the Direct Monte Carlo method and can save a lot of computation time at the same time, it is both accurate and efficient, and is very competitive to be used in structural reliability analysis.
\end{abstract}

\section{Introduction}

Bridge is the hinge of transportation system and the important component of lifeline project, and the safety of the bridges is directly related to the national economy and people's livelihood. Thus, conducting structural reliability analysis is in general an important task, which has aroused the interest of many scholars in recent years.

The failure probability is not only a key indicator to measure structural safety, but also a basic research area in structural reliability analyses. Since multiple probability factors existed in the safety analysis of structural component or system, a multi-fold integral needs to be calculated to obtain the failure probability of the structure. However, even if the performance function is explicit, the nonlinearity of the function and the non-Gaussian distribution of random variables always make it impossible to obtain the exact solution of this multi-fold integral [1], [2]. Various methods have been used to analyze the failure probability of structures subjected to random material parameters, such as the first- and second- order reliability methods (FORM/SORM) [3], [4], the simulation methods [5]-[7], the traditional perturbation stochastic finite element methods (PSFEM) [8], [9], the response surface method [10], [11], and so on. However, more or less problems exist in the above mentioned methods. For example, FORM/SORM are ineffective when response sensitivities are not available, the limit state function is highly nonlinear or multiple MPPs exsit. The most representative and effective simulation method is the Direct Monte Carlo simulation (DMC) [12], which is usually used to verify the accuracy of the other methods, but it is also very time-consuming which restricts its application in structural safety analysis, especially in large and complex structures. PSFEM is widely used for stochastic series expansion, but the random responses of structures may be unstable if the uncertainty of some random parameters is large. The response surface method is a good choice when some specific types of multivariate polynomial are assumed to represent the response surface of structure, but further research is needed to ensure the correctness of the assumed form of the response surface.

In this study, an innovative method is presented to analyze the failure probability of structures which is mainly based on an improvement strategy of high order perturbation stochastic finite element method [13], 14]. Afterwards, in terms of an orthogonalization technique, the initial series solution is transferred to be a set of orthogonalizable power polynomial basis vectors. Through the usage of Galerkin projection scheme, an accelerating factor vector of the orthogonalizable power polynomial expansion is determined by solving small scale algebraic equations. Due to the acquisition of the explicit expression of random displacement response, the DMC method is then used to evaluate the failure probability directly and quickly. Finally, a numerical example is provided to illustrate the efficiency and accuracy of the proposed method (OSFEM-P).

\section{The OSFEM-P Method}

\subsection{Random static equilibrium equation}


When small deformation of a linear elastic structural system occurs under an load, the equation can be expressed as the following

$$
\mathbf{K d}=\mathbf{f}
$$

where $\mathbf{K}$ is the structural stiffness matrix; $\mathbf{f}$ is equivalent nodal load vector.

The uncertain stiffness matrix can be expressed as

$$
\mathbf{K}=\mathbf{K}_{0}+\sum_{i=1}^{n_{1}} \alpha_{i} \mathbf{K}_{i}
$$

where $\mathbf{K}_{0}$ is a $N \times N$ dimensional deterministic matrix with respect to deterministic mean parameters. $\mathbf{K}_{i}$ is a $N \times N$ dimensional matrix.

When external load includes $l$ random variables, it can be written as

$$
\mathbf{f}=\mathbf{f}_{0}+\sum_{i=n_{1}+1}^{n} \mathbf{f}_{i} \alpha_{i} \quad\left(n=n_{1}+l\right)
$$

where $\mathbf{f}_{0}$ is a $N \times 1$ dimensional deterministic vector with respect to deterministic mean parameters and $\mathbf{f}_{i}$ is a $N \times 1$ dimensional vector.

The random response vector $\mathbf{d}$ is used by the following multivariate power series expansion as

$$
\begin{aligned}
& \mathbf{d}(\mathbf{X}, \boldsymbol{\alpha})=\mathbf{d}_{0}(\mathbf{X}) \varphi_{0}+\sum_{i=1}^{\infty} \mathbf{d}_{i}(\mathbf{X}) \varphi_{1}(\boldsymbol{\alpha})+ \\
& \sum_{i=1}^{\infty} \sum_{j=1}^{i} \mathbf{d}_{i j}(\mathbf{X}) \varphi_{2}(\boldsymbol{\alpha})+\sum_{i=1}^{\infty} \sum_{j=1}^{i} \sum_{k=1}^{j} \mathbf{d}_{i j k}(\mathbf{X}) \varphi_{3}(\boldsymbol{\alpha})+\cdots
\end{aligned}
$$

where $\varphi_{i}(\boldsymbol{\alpha})$ denotes the multidimensional power series, the first four terms can be written as

$$
\varphi_{0}(\boldsymbol{\alpha})=1 ; \varphi_{1}(\boldsymbol{\alpha})=\alpha_{i} ; \varphi_{2}(\boldsymbol{\alpha})=\alpha_{i} \alpha_{j} ; \varphi_{3}(\boldsymbol{\alpha})=\alpha_{i} \alpha_{j} \alpha_{k}
$$

\subsection{Perturbation technique}

Substituting (2-4) into (1) results in the following

$$
\left(\mathbf{K}_{0}+\sum_{i=1}^{n} \alpha_{i} \mathbf{K}_{i}\right)\left[\mathbf{d}_{0} \varphi_{0}+\sum_{i=1}^{n} \mathbf{d}_{i} \varphi_{1}+\cdots\right]=\mathbf{f}_{0}+\sum_{i=1}^{n} \mathbf{f}_{i} \alpha_{i}
$$

where $\mathbf{d}$ is defined as a truncated series which includes $n$ random variables at the same time, and

$$
\mathbf{K}_{i}=0\left(i=n_{1}+1, \cdots n\right) ; \quad \mathbf{f}_{i}=0\left(i=1, \cdots n_{1}\right)
$$

Since every sample of random vector $\boldsymbol{\alpha}$ should satisfy (6), then the sum of coefficients of the same order terms based on power series expansion should be equal to zero. Therefore, the coefficient vectors of the power series expansion can be determined by perturbation technique.

\subsection{Orthogonalizable technology and Galerkin projection scheme}

In general, the convergent rate for the power series expansion is not often satisfied, thus, Galerkin projection is taken as an improvement measure.

Before utilizing that scheme, $\mathbf{d}$ can be transferred into an orthogonal polynomial expansion $\overline{\mathbf{d}}$ by using a transformation matrix in order to use Galerkin projection scheme more easily, then

$$
\overline{\mathbf{d}}=\overline{\mathbf{d}}_{0} \chi_{0}+\sum_{i=1}^{n} \overline{\mathbf{d}}_{i} \chi_{1}+\sum_{i=1}^{n} \sum_{j=1}^{i} \overline{\mathbf{d}}_{i j} \chi_{2}+\cdots
$$

where $\chi_{0}, \chi_{1}, \chi_{2} \cdots$ are orthogonal basis vectors.

If correction factors are employed in each term in (8), then

$\overline{\mathbf{d}}=\beta_{0} \overline{\mathbf{d}}_{0} \chi_{0}+\beta_{1} \sum_{i=1}^{n} \overline{\mathbf{d}}_{i} \chi_{1}+\beta_{2} \sum_{i=1}^{n} \sum_{j=1}^{i} \overline{\mathbf{d}}_{i j} \chi_{2}+\cdots=\sum_{i=0}^{m} \beta_{i} \psi_{i}$

where $\psi_{0}=\overline{\mathbf{d}}_{0} \chi_{0} ; \psi_{1}=\sum_{i=1}^{n} \overline{\mathbf{d}}_{i} \chi_{1} ; \psi_{2}=\sum_{i=1}^{n} \sum_{j=1}^{i} \overline{\mathbf{d}}_{i j} \chi_{2}$ and so on, $\beta_{0}, \beta_{1} \cdots \beta_{m}$ are correction factors.

By using Galerkin projection scheme, (9) can be written as following

$$
\sum_{j=0}^{m} \sum_{i=0}^{n}<\alpha_{i}\left(\psi_{k}\right)^{T} \mathbf{K}_{i} \psi_{j}>\beta_{j}=\sum_{i=0}^{n}<\alpha_{i}\left(\psi_{k}\right)^{T} \mathbf{f}_{i}>
$$

where $k=0, \cdots m, \quad<\cdot>$ denotes the operator of mathematical expectation.

By solving the small scale $(\mathrm{m}+1) \times(\mathrm{m}+1)$ linear algebraic equations, the unknown coefficients $\beta_{0}, \beta_{1}$ $\cdots \beta_{m}$ can be easily determined. Then, the random response vector is capable of being got.

\section{Reliability analysis}

\subsection{Failure probability}

According to the definition of the failure probability $P_{F}$, it can be determined by direct integration over the failure domain as follows

$$
\begin{aligned}
& P_{F}=P(\Psi(\boldsymbol{\alpha})<0) \\
& =\int_{\Psi(\boldsymbol{\alpha})<0} f_{p}\left(\alpha_{1}, \alpha_{2}, \cdots, \alpha_{n}\right) d \alpha_{1} d \alpha_{2} \cdots d \alpha_{n}
\end{aligned}
$$

where $f_{p}\left(\alpha_{1}, \alpha_{2}, \cdots, \alpha_{n}\right)$ is the joint probability distribution function of the random variables $\left\{\alpha_{1}, \alpha_{2}, \cdots, \alpha_{n}\right\}$ and $(\Psi(\boldsymbol{\alpha})<0)$ defines structural failure.

\subsection{Calculation of multifold Integral}

Generally, even if the explicit expressions of random structural responses are determined, it is still difficult to calculate the exact failure probability due to the reason that the failure domain of numerical interval is difficult to obtain or the explicit performance function is complex. 
If the partial derivative of performance function is given, FORM, SORM and other similar methods can be used to calculate the failure probability. However, results based on FORM/SORM should be interpreted with caution for highly nonlinear performance functions or multiple design point, which exists in many structural systems.

Since the random response vector is obtained, so the response surface is apparent.

$$
P\left(\boldsymbol{\alpha}^{(i)}\right)=\Theta-\mathbf{d}\left(\boldsymbol{\alpha}^{(i)}\right)
$$

where $\boldsymbol{\alpha}^{(i)}$ is the $i$ th sample of the random vector $\boldsymbol{\alpha} ; \Theta$ denotes the threshold value of displacement at some point of the structural system; $\mathbf{d}(\boldsymbol{\alpha})$ is the displacement response vector of the structural system under external loads.

The Monte Carlo simulation is used to calculate the failure probability directly.

$$
P_{F}=\frac{1}{N} \sum_{i=1}^{N} \mathrm{H}\left[P\left(\boldsymbol{\alpha}^{(i)}\right)<0\right]
$$

where $N$ is the sample size; $\mathrm{H}$ is defined as counting function; if $P\left(\boldsymbol{\alpha}^{(i)}\right)$ is in failure area, then $\mathrm{H}=1$.

\section{Numerical example}

Considering a continuous beam type of bridge structure with two spans, which can be simplified into a onedimensional continuous beam subjected to a concentrated force, as shown in Fig. 1. The whole length of the beam is $8 m$, and the beam is divided into 16 elements. Assuming that the bending rigidity $E I$ of the beam and the external load $F$ are both random variables. The means of $E I$ and $F$ are $3.4 \times 10^{4} \mathrm{kN} \cdot \mathrm{m}^{2}$ and $40 \mathrm{kN}$ respectively.

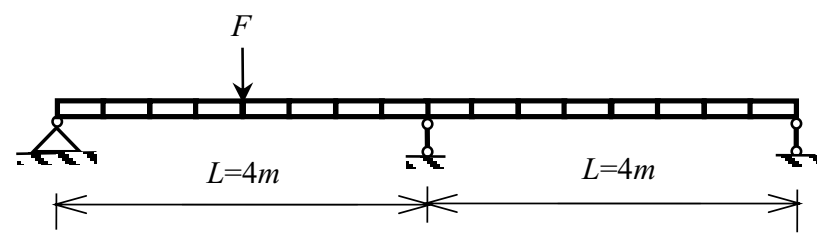

Figure 1. Random continuous beam

Assuming that $E I$ and $F$ are both of Beta distribution, thus we have

$$
E I=E I_{0}\left(1+\sqrt{5} \cdot \alpha_{1} \cdot \delta_{1}\right) ; F=F_{0}\left(1+\sqrt{5} \cdot \alpha_{2} \cdot \delta_{2}\right)
$$

where $\mathrm{EI}_{0}$ and $\mathrm{F}_{0}$ denote the means of $E I$ and $F$ respectively; $\delta_{E I}, \delta_{F}$ are the coefficients of variation (COVs) of $E I$ and $F$ respectively. In addition, $\alpha_{i}(i=1,2)$ are independent random variables with Beta distribution, of which the probability density function is written as

$$
f(\alpha)=\frac{3}{4}\left(1-\alpha^{2}\right) \quad \alpha \in(-1,1)
$$

Here $\mathbf{d}\left(\boldsymbol{\alpha}^{(i)}\right)$ denotes the vertical displacement at the middle of the left span of the beam. The failure probability of the random beam is computed by four methods, i.e., FORM, SORM, OSFEM-P, DMC. the results of failure probability calculated by these four methods are listed in Table 1.

Here it is assumed that $\delta_{F}$ is equal to 0.1 and $\delta_{E I}$ is supposed to be $0.1,0.15,0.2,0.25$ and 0.3 respectively. The threshold value, $\Theta$, of the vertical displacement at the middle of the left span of the beam is assigned as $1.702 \times 10^{-3} \mathrm{~m}$.

Table 1 shows the results of OSFEM-P are the most close to that of DMC, and SORM is more precise than FORM, especially when the $\delta_{E I}$ is small.

Table 1. The failure probability of the beam from different methods with $\delta_{F}=0.1$

\begin{tabular}{|c|c|c|c|c|c|}
\hline \multirow{2}{*}{ Methods } & \multicolumn{5}{|c|}{$\delta_{E I}$} \\
\cline { 2 - 6 } & 0.1 & 0.15 & 0.2 & 0.25 & 0.3 \\
\hline FORM & $8.40 \mathrm{e}-4$ & $1.80 \mathrm{e}-2$ & $6.37 \mathrm{e}-2$ & $1.16 \mathrm{e}-1$ & $1.63 \mathrm{e}-1$ \\
\hline SORM & $5.62 \mathrm{e}-4$ & $1.33 \mathrm{e}-2$ & $5.60 \mathrm{e}-2$ & $1.10 \mathrm{e}-1$ & $1.59 \mathrm{e}-1$ \\
\hline OSFEM-P & $1.00 \mathrm{e}-4$ & $1.31 \mathrm{e}-2$ & $5.43 \mathrm{e}-2$ & $1.09 \mathrm{e}-1$ & $1.60 \mathrm{e}-1$ \\
\hline DMC & $1.00 \mathrm{e}-4$ & $1.31 \mathrm{e}-2$ & $5.41 \mathrm{e}-2$ & $1.09 \mathrm{e}-1$ & $1.60 \mathrm{e}-1$ \\
\hline
\end{tabular}

The spent computation time of the four methods are also recorded and listed in Table 2, it can be seen that the DMC method, the highest accurate method, is the most time-consuming method, which is hundreds times of the proposed method and the widely used FORM and SORM. The time cost of the proposed method is very close to the FORM and SORM, but it can significantly increase the computation accuracy. Thus, the proposed method is both accurate and efficient in structural reliability analysis.

Table 2. CPU time spent of different methods.

\begin{tabular}{|c|c|c|c|c|}
\hline Methods & DMC & OSFEM-P & FORM & SORM \\
\hline CPU times (s) & 1326.4 & 3.2 & 1.2 & 1.5 \\
\hline
\end{tabular}

To fully illustrate the effects of the random inputs on the statistical properties of random response, the curves of probability density function (PDF) of the vertical displacement at the middle of the left span of the beam with the proposed method is shown in Fig. 2 and Fig. 3. It can be seen that the PDF curves of the vertical displacement at the middle of the left span of the beam vary with different $\delta_{E I}$ or $\delta_{F}$, herein, we choose the two cases as following:

Case 1: smallest failure probability in Table 1, $\delta_{E I}=0.1$ with $\delta_{F}=0.1$

Case 2: largest failure probability in Table 1, $\delta_{E I}=0.3$ with $\delta_{F}=0.1$ 


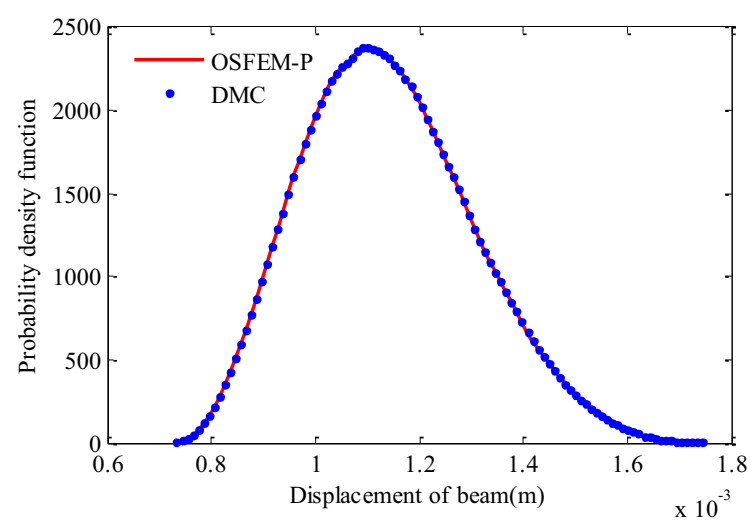

Figure 2. PDF from DMC and proposed method (case 1).

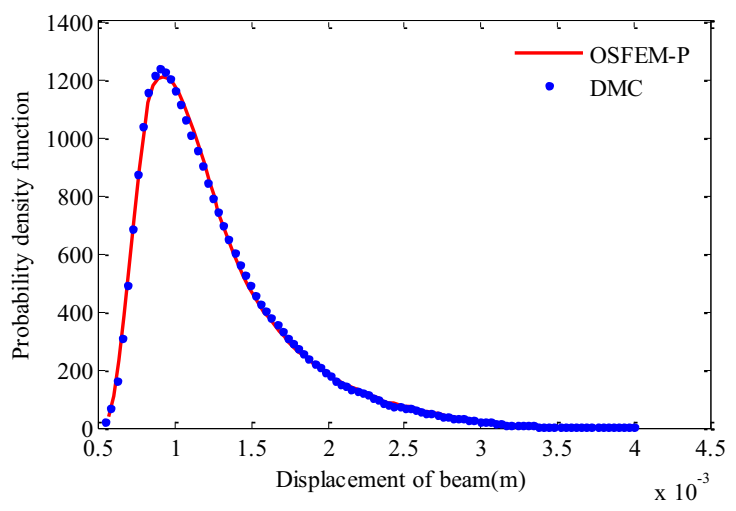

Figure 3. PDF from DMC and proposed method (case 2).

Fig. 2 and Fig. 3 show that the output displacements of the beam are diverse from each other for different $\delta_{E I}$, and the PDF curves of OSFEM-P are very close to that of DMC no matter $\delta_{E I}$ is small or large, which illustrates the accuracy of the proposed OSFEM-P is very good.

\section{Conclusions}

In this study, a new method, i.e. OSFEM-P, for structural reliability analysis based on orthogonalizable power polynomial basis vector is presented. Firstly, a power polynomial basis vector is adopted to express the initial series solution of structural response, which is determined by a series of deterministic recursive equation based on perturbation technique, and then transferred to be a set of orthogonalizable power polynomial basis vector using the orthogonalization technique. By conducting Garlekin projection, an accelerating factor vector of the orthogonal power polynomial expansion is determined by solving small scale algebraic equations. Numerical results of a continuous bridge structure shows that the proposed method is both accurate and efficient, it can significantly improve the calculation accuracy compared with the widely used FORM/SORM and save a lot of computation time compared with the DMC method.
Financial support from the National Nature Science Foundation of China under Project No. 51378407 and 51578431 is gratefully acknowledged.

\section{References}

1. K. Breitung. Asymptotic approximations for multinormal integrals. J Eng Mech, 110(3): 357-366 (1984).

2. G.Q. Cai and I. Elishakoff. Refined second-order reliability analysis. STRUCT SAF, 14(4): 267-276 (1994).

3. M.A. Valdebenito, H.J. Pradlwarter, G.I. Schuëller. The role of the design point for calculating failure probabilities in view of dimensionality and structural nonlinearities. Struct SAF, 32(2):101-111 (2010)

4. R. Rackwitz. Reliability analysis - a review and some perspectives. Struct SAF, 23(4): 365-395 (2001).

5. G.I. Schuëller, H.J. Pradlwarter, P.S. Koutsourelakis, A critical appraisal of reliability estimation procedures for high dimensions. Probabilist Eng Mech. 19(4): 463-474 (2004).

6. S.K. Au and J.L. Beck. Estimation of small failure probabilities in high dimensions by subset simulation. Probabilist Eng Mech, 16(4): 263-277 (2001).

7. G.I. Schuëller, H.J. Pradlwarter and P.S.Koutsourelakis. A critical appraisal of reliability estimation procedures for high dimensions. Probabilist Eng Mech, 19(4): 463-474 (2004).

8. J.D. Arregui-Mena, L. Margetts, P.M. Mummery. Practical Application of the Stochastic Finite Element Method. Arch Comput Method E, 23(1):171-190 (2014).

9. B. Huang, Q.S. Li and W.H. Shi, Z. Wu. Eigenvalues of structures with uncertain elastic boundary restraints. Appl Acoust, 68(3): 350-363 (2007).

10. N. Balakrishnan, M.H. Ling. Gamma lifetimes and one-shot device testing analysis. Reliab Eng Syst Safe, 126(6), 54-64 (2014).

11. X.L. Guan and R.E.Melchers. Effect of response surface parameter variation on structural reliability estimates. Struct Saf, 23(4): 429-444 (2001).

12. R.Y. Rubinstein. Simulation and the Monte Carlo method. Wiley, New York, America (1981).

13. B. Huang, Q.S. Li, A.Y. Tuan and H. Zhu. Recursive approach for random response analysis using nonorthogonal polynomial expansion. Comput Mech, 44(3): 309-320 (2009).

14. B. Huang, R.F. Seresh and L. Zhu. Statistical analysis of basic dynamic characteristics of large span cable-stayed bridge based on high order perturbation stochastic FEM. Adv Struct Eng, 16(9): 1499-1512 (2013). 